\title{
Third telescope project at the IOTA interferometer
}

\author{
Wesley A. Traub, Nathaniel P. Carleton, Jesse D. \\ Bregman, Michael K. Brewer, Marc G. Lacasse, et al.
}

Wesley A. Traub, Nathaniel P. Carleton, Jesse D. Bregman, Michael K. Brewer, Marc G. Lacasse, P. Maymounkov, Rafael Millan-Gabet, John D. Monnier, Sebastien Morel, Costas D. Papaliolios, Michael R. Pearlman, Irene L. Porro, F. Peter Schloerb, Kamal Souccar, "Third telescope project at the IOTA interferometer," Proc. SPIE 4006, Interferometry in Optical Astronomy, (5 July 2000); doi: 10.1117/12.390273

SPE. Event: Astronomical Telescopes and Instrumentation, 2000, Munich, Germany 


\title{
The Third Telescope Project at the IOTA Interferometer
}

\author{
Wesley A. Traub ${ }^{a}$, N.P. Carleton ${ }^{a}$, J.D. Bregman ${ }^{b}$, M.K. Brewer ${ }^{c}$, M.G. Lacasse ${ }^{a}$, \\ P. Maymounkov ${ }^{a}$, R. Millan-Gabet ${ }^{a}$, J.D. Monnier ${ }^{a}$, S. Morel $^{a}$, C. Papaliolios ${ }^{a}$, \\ M.R. Pearlman ${ }^{a}$, I.L. Porro ${ }^{a}$, F.P. Schloerb ${ }^{c}$, K. Souccar ${ }^{c}$ \\ ${ }^{a}$ Harvard-Smithsonian CfA, 60 Garden St., Cambridge, MA 02138 \\ ${ }^{b}$ NASA Ames Research Center, MS 245-6, Moffett Field, CA 94035 \\ ${ }^{c}$ Physics and Astronomy Dept., Univ. of Massachusetts at Amherst, Amherst, MA 01003
}

\begin{abstract}
The third telescope project to enable phase-closure observations at the IOTA interferometer is well underway, and is anticipated to be completed later this year. For this project, we present the main technical improvements which we have already made or expect to make, including a new VxWorks control system, improved star acquisition cameras, improved siderostat and primary mirror supports, five-axis control of the telescope secondary mirrors, automated control of the long delay line, trihedral retroreflectors, three-beam combination, the PICNIC camera, and fringe packet tracking.
\end{abstract}

Keywords: Interferometer, IOTA

\section{INTRODUCTION}

The Infrared Optical Telescope Array (IOTA) began scheduled operation in 1995, with two $0.45 \mathrm{~m}$ telescopes, and baselines up to $38 \mathrm{~m}$. At the present time (1999-2000), a 3rd telescope and siderostat have been installed at IOTA, and the corresponding optics have all been polished and silver coated, and are either installed or nearly so. Likewise, the relay optics are mounted and ready to be installed. Three-beam operation is planned for late 2000 .

An overview of progress and scientific results through 1998 was given in Traub. ${ }^{1}$ Current progress and further scientific results are given elsewhere in this volume by Berger, ${ }^{2}$ Hofmann, ${ }^{3}$ Kervella, ${ }^{4}$ Mennesson, ${ }^{5}$ Millan-Gabet, ${ }^{6}$ and Morel. $^{7}$

In the present paper we outline some of the key projects currently underway at IOTA, in addition to those just mentioned above. The results here are essentially a progress report, since most of these projects are still in development.

\section{VXWORKS CONTROL SYSTEM}

\subsection{Introduction}

The goal of a telescope Monitor and Control system is to provide an efficient computing environment for monitoring, control, and data acquisition. The system should provide users with tools to compose observing plans, to submit them for execution, to monitor their execution in real time, to monitor the data generated by the instruments in real time, and to record and archive scientific and engineering data. The system should also provide interfaces to integrate new instruments and to host visiting instruments; and it must be capable of incorporating user preferences.

The basic architecture and design principles for the IOTA Monitor and Control system are based on work being carried out in support of the Large Millimeter Telescope (LMT) project at the University of Massachusetts. IOTA is making use of the LMT development effort, which is designing an open architecture system that is implemented using existing components developed at other observatories wherever possible.

Further author information: (Send correspondence to W. Traub).

Email: WAT: wtraub@cfa.harvard.edu ; NPC: ncarleton@cfa.harvard.edu ; JDB: jbregman@mail.arc.nasa.gov ;

MKB: brewer@fcraol.phast.umass.edu ; MGL: mlacasse@cfa.harvard.edu ; PM: maymounk@fas.harvard.edu ;

RMG: rmillan@cfa.harvard.edu ; JDM: jmonnier@cfa.harvard.edu ; SM: smorel@cfa.harvard.edu ;

CP: cpapaliolios@cfa.harvard.edu ; MRP: mpearlman@cfa.harvard.edu ; ILP: iporro@cfa.harvard.edu ;

FPS: schloerb@astro.umass.edu ; KS: souccar@astro.umass.edu 


\subsection{Description of the Monitor and Control System}

The IOTA monitor and control system comprises of a set of modules with control and data flows among them. It is illustrated in Figure 1. At the front end of the system is an observing tool that is used to compose observing plans. These plans are in turn submitted to a finite state machine controller to be executed according to a predefined or dynamically determined schedule. The same observing tool is used to run the telescope interactively for scientific or engineering purposes.

While observing commands are being executed, the state of the system is monitored by the user, and scientific and engineering data are collected. An observing simulator is provided to test the validity of observing programs.

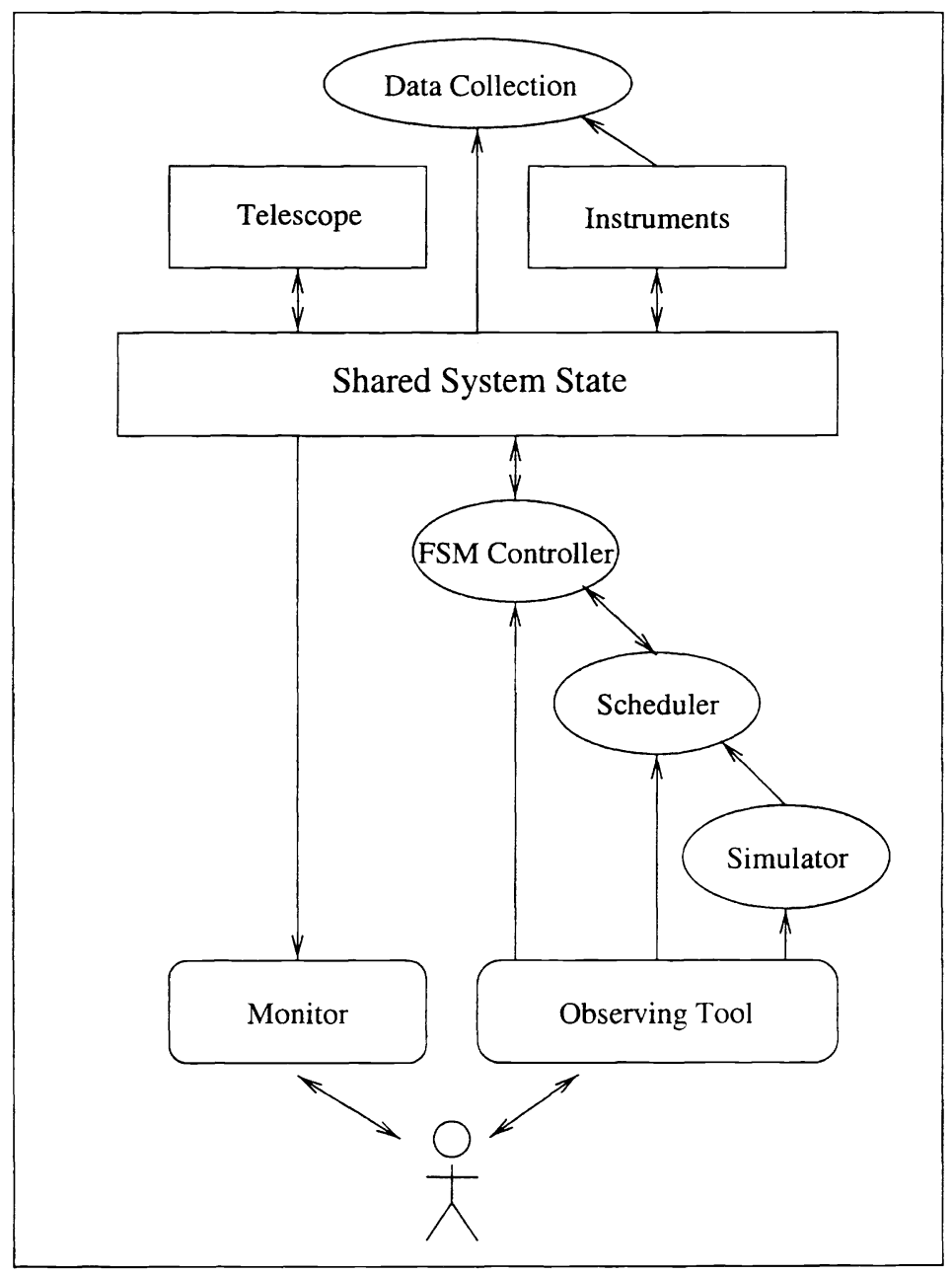

Figure 1. The IOTA Monitor and Control System

\subsubsection{Observing Tool}

The observing tool provides the means for the user to input desired commands to the system and to execute them on line. These commands can also be saved in a file to be scheduled for later execution.

The user creates a science program that may include one or more observing programs. Each observing program must contain scheduling constraints, target positions, a science instrument, and a data collection method.

In turn, the observing tool generates commands to control the different subsystems based on the science program composed by the user. The generated commands are implemented using a keyword-value pair method to set the 
parameters of each subsystem. Expert users may choose to generate the observing commands by hand to short cut this process.

Ultimately, the project would like to modify Gemini's observing tool to be used for the IOTA and the LMT. In the mean time, we have built a web-based tool that mimics the functionality of Gemini project's observing tool to define science programs.

\subsubsection{Scheduler}

A queue scheduler schedules the execution of science programs according to their priorities and meteorological conditions. However, this does not preclude the system from accepting direct commands when it is in manual scheduling mode.

\subsubsection{FSM Controller}

A finite state machine (FSM) controller translates the observing programs into desired system states. It steps through the science program and executes each command while monitoring the state of the system.

\subsubsection{Monitoring}

The purpose of on-line system monitoring is to provide a running check on data integrity and proper system operation by displaying the state of the system to the user. The monitoring system provides real-time access to the observation state, telescope state, instrument states, etc. The initial implementation of the monitoring system uses Java software developed at the Owens Valley Radio Observatory by Steve Scott.

\subsubsection{Data Collection and Processing}

Data collection is the final outcome of an observing program. Data from instruments is tagged with telescope and system states and saved to disk for further processing. A quick-look data display, and some degree of quick analysis, should be provided.

\subsubsection{Observing Simulator}

The observing simulator is designed to test observing programs and train and familiarize observers with the telescope system. It provides the same exact functionality as the real system and can be run in real time, faster than real time, or single stepped through a debugging program.

\subsection{Architecture of the Monitor and Control System}

The complexity and requirements of the Large Millimeter Telescope necessitate a real-time distributed processing approach. Therefore, the LMT monitor and control system is being implemented as a distributed system consisting of independent modules running on multiple computers. The LMT Project has selected replicated shared memory to provide the interconnection among the multiple processes running on the different computing systems. This choice eliminates the large overhead associated with inter-process communication and minimizes real-time communication dependencies between subsystems. The initial implementation of the IOTA system will be far simpler than the LMT system and will probably be handled by one or more processors using shared memory in a single VME crate to provide the interconnection among the multiple processes running on the system.

\section{IMPROVED STAR ACQUISITION CAMERAS}

Each telescope at IOTA has a commercial CCD camera mounted near the back of the primary mirror, pointing perpendicular to the telescope axis, and illuminated by the 10-times reduced telescope beam from the secondary mirror via a remotely-controlled flip-in mirror. We recently installed new cameras which are an improvement over our previous ones in two aspects: the sensitivity is better, and the integration time can be selected. The new cameras are Panasonic WV-BP-550 black and white CCD cameras. The lens is a Fujinon f/1.2 zoom lens, which we set to its maximum focal length of $75 \mathrm{~mm}$. This combination gives approximately 2 arcsec pixels on the sky, and about 15 arcmin field of view.

We also installed a Panasonic "looping switch" box and a controller, giving us the ability to display up-to 8 camera inputs, either one at a time on a single monitor, or simultaneously on 1 monitor per camera. The controller 
has many other functions, but the one which is most important to us is the ability to control the integration time, from a normal fast framing rate of $30 \mathrm{frames} / \mathrm{sec}$, to a slow rate of about $1 \mathrm{frame} / \mathrm{sec}$, in 6 selectable steps.

At the normal rate we can now easily see a $V=12$ magnitude star, whereas with our previous cameras a $V=12$ star was only barely visible against the background noise. Slowing the frame rate allows even fainter stars to be seen easily, but we find that the normal rate is adequate and comfortable for almost all our current work.

\section{IMPROVED SIDEROSTAT AND PRIMARY MIRROR SUPPORT}

In a recent study of the optical quality of the IOTA system ${ }^{8}$ we found that the measured visibilities in the visible and infrared were approximately consistent with the known sources of wavefront degradation. Ranking the expected main sources of wavefront degradation, we found that 3 of them dominated: the wavefront curvature from atmospheric turbulence, the servo system time constant, and the flatness of the relay optics surfaces, approximately in rank order.

We intend to address the first 2 sources in the future. However we recently attempted to reduce a part of the flatness error by improving the mounting technique used for the 2 large optical elements in our train, the siderostat and the telescope primary mirror. These are approximately $45 \times 68 \mathrm{~cm}$ and $45 \mathrm{~cm}$ diameter, respectively, and each is about $7.6 \mathrm{~cm}$ thick, i.e., about $1 / 6$ times the beam diameter.

The primary mirror points downward toward the siderostat mirror, with its optical axis inclined by $30^{\circ}$ below the horizontal. Most of the weight of the mirror is supported by 2 nominally low-friction pads along the bottom edge of the mirror. There are also 3 hard points on the back of the mirror, and each is flanked closely by 2 springs which pull the mirror back against the hard points.

The improvement we have recently made is to incorporate high precision ball slides into the 2 lower edge supports to reduce friction. The friction is now small enough that the springs on the back of the mirror easily keep the mirror held snugly against its 3 back-plane hard points. Previously, the edge friction force was sometimes greater than this spring force, and the mirror would occasionally slip away from its lower hard point, thereby misaligning the telescope slightly. The larger edge friction also could have contributed to a bending of the mirror, which we are now sure is negligibly small.

We also installed a lifting spring on each primary mirror, pulling upward at a $30^{\circ}$ angle from the vertical, on the top edge of the mirror, along a line perpendicular to the optical axis. This spring is gauged to oppose the tendancy of the top edge of the mirror to droop forwards.

The siderostat flat rests on 3 hard points on its back side, and is restrained from slipping in the plane of the mirror surface by a pair of tangent arms. The original design included 6 air bags on the back of each siderostat mirror, which could be inflated with air and which would then remove some of the weight of the mirror from the 3 hard points. However we found it difficult to control the air pressure sufficiently well to provide a nominal lifting force, without floating the mirror completely free of the hard points, so in practice we have not used the air bag system. However we recently purchased a custom air bag which covers much of the back area of the mirror, and will more easily take up the irregularities of spacing between the mirror and the cradle back. We expect to install and test this device in the near future.

\section{FIVE-AXIS CONTROL OF THE TELESCOPE SECONDARY MIRRORS}

The original design of the IOTA secondary mirror mounts included a 3-axis translation stage immediately behind the secondary mirror, adjustable by manual micrometer screws, and a tip-tilt adjustment capability of the entire spider assembly via coarse screw threads at the ends of the spider arms. Aside from this adjustment capability, the mechanical spacing of the primary and secondary has been, and will continue to be, controlled through a path that is nominally thermally invariant, using 3 ceramic rods and appropriate metal connection.

However this original mechanism of adjustment of the secondary with respect to the primary proved to be awkward in practice, in part because the tip-tilt also had a component of translation, and in part because the adjustments were done manually at the telescope, whereas the sensing of the wavefront was done interferometrically in the laboratory, making communication between the people at either end difficult.

We recently made 2 major changes to this system, with the hope of making this adjustment easier to carry _out.

The first change is that we replaced the secondary mirror's commercial 3-axis translation stage with a compact 5 -axis stage of our own design. The new stage uses New Focus Picomotors for motion control. These devices have 
step sizes of about $1 / 40$ wavelength, and they are remotely commandable. Three Picomotors are mounted directly behind the secondary mirror, with their axes nominally parallel to the telescope optical axis. Thus a motion of any one of these motors produces a tip or tilt action of the secondary, plus a small translation. The action of all 3 together produces a focus change. In our design these 3 motors are mounted on a plate which can be rotated slightly about 2 axes perpendicular to the optical axis, thus producing a translation of the secondary, perpendicular to the focus direction. The pivot axes are supported by flexure elements from Lucas Aerospace. The combination gives complete 5-axis control.

After building these units, and permanently installing them on the telescopes, we discovered 2 problems which we are still working to resolve. One problem is that the motors have significantly different step sizes from motor to motor, due in part to the different axial loading forces on each screw, caused by differences in tension of the pre-load springs used in our design. A second problem is that each motor has a significantly different step size in the forward and reverse direction, a factor which seems to be independent of axial load or friction.

(We also discovered a third problem, which we have since solved. In an effort to avoid several mechanical interferences, we removed the knurled knob attached to the end of several of our Picomotors. The knob is used to manually turn the Picomotor screw, and we thought we could work without it present. It turns out that the inertia of the knob is crucial to the successful operation of a Picomotor, and to restore nominal operability we had to replace the knobs with little flywheels of comparable inertia, tailored to our spatial constraints.)

From our limited experience it seems that the individual step size differences can probably be calibrated to some extent, but we have not yet done this. Calibration will be required in order to make our 5-axis stages easier to use. An additional step will then be to write the remote command program in such a way that it can carry out combined motions of the motors to give focus, orthogonal translation, and tip-tilt.

The second change was to build a simple telescope alignment interferometer for use at the telescope, instead of having the interferometer in the distant laboratory. The advantage here is simply that the distance from the secondary to the test interferometer is now only about $3 \mathrm{~m}$, as opposed to about $30 \mathrm{~m}$ to the lab, so that a small change of the secondary mirror tilt or position will not cause the reflected laser beam to become lost. The system works by sending an expanded parallel beam of laser light out from the lab, passing it through a beamsplitter near the telescope, sending the reflected beam to a flat mirror and back to the beamsplitter, and sending the transmitted beam to the secondary, primary, siderostat flat (tilted to autocollimation position), back to the primary, to the secondary, and finally to the beamsplitter. Fringes in the combined output beam indicate telescope misalignment. We adjust the secondary to eliminate the fringes, as much as possible. Residual errors amount to roughly one fringe, due in part to flatness errors, and perhaps in part to mounting errors in the large optics, as discussed above. Operationally, the fringes are easy to see by eye, and the adjustment of the secondary is relatively rapid, although due to the problems mentioned above with motor step sizes, at present we are making these adjustments by manually turning the motor screws, which is adequate from a sensitivity point of view.

\section{AUTOMATED CONTROL OF THE LONG DELAY LINE}

In the original design of IOTA, we split the beam from a single laser to control both the short and long delay lines, which sufficed for a 2-telescope interferometer. In the current design, we have 3 telescopes, and must therefore have at least 2 sets of short and long delay lines. An additional requirement is that we want to have the long delay position continuously monitored, whereas in the original system we shuttered the long delay laser beam during star observations, because it scattered light into the science beam.

The new design uses one laser split to control the 2 short delay lines, and a second laser split to control the 2 long delay lines. In addition, the long delay laser has been moved to the far end of the delay line, so that the laser beam no longer runs close to the science beam. The laser retroreflector has been moved from the front of each long delay cart to the back of the cart. Fine control of the laser beam direction is controlled by a steering flat mounted on a 2-axis Picomotor mount, which gives adequate directional control.

The long delay laser is an HP-5517A. We also use HP optics, receiver heads, and pulse counting electronics boards, the latter being installed in a dedicated computer. The laser and computer are located in a small hut at the extreme north-east end of the long delay line. The laser beams are passed into the vacuum tank via 2 windows mounted in the end cap of the long delay vacuum pipe. 
The remote computer communicates via UDP packets, once per second, in a client-server relationship, with a control computer in the laboratory. Several modes of operation are available. The long delay cart can be commanded to home, using an opto-interrupter switch, giving an accuracy of about $10 \mu \mathrm{m}$, which is adequate. The cart can be moved to a desired position within $1 \mathrm{~mm}$ or so, using open-loop commands to a stepping motor that drives a steel cable pulling the cart. The actual position achieved is determined from the laser, and the offset from the desired position is communicated to the short delay line, which compensates accordingly.

The cart has a slew speed of $20 \mathrm{~cm} / \mathrm{sec}$, which means that long delay reconfigurations can be carried out relatively quickly. Since the position is continuously monitored, we have the capability to correct for small thermal changes throughout the night.

The new system is thus a big improvement over the original system, which required a sequence of relatively clumsy and slow operations each time the cart needed to be moved. The only remaining issues which we need to address in the near future are the straightness of the track, which causes the laser beam to lose strength (but not to fail) at one particular segment of the track, and the scattered laser light, which needs some basic baffling to keep it from leaking past the cart and into the vicinity of the science beam.

\section{TRIHEDRAL RETROREFLECTORS}

The original IOTA design uses dihedral (2 flats at $90^{\circ}$ ) reflectors on both the long and short delay lines. About one year ago we began to fabricate trihedral ( 3 flats at $90^{\circ}$ to each other) reflectors, also known as hollow cube corners, to replace the dihedrals.

Our reason for wanting trihedrals is that they are immune to tilt errors. Our long delay line track is straight to an accuracy of only about $0.01 \mathrm{~cm}$ or so, which means that every time the dihedral is moved to a new position on the track, the dihedral must also be corrected in yaw angle by roughly 100 arcsec. We routinely do this operation manually, taking as our visual reference a Maglite bulb which is flipped onto the nominal optical axis near the telescope. We then send a modulated infrared signal to a battery-driven motor on the cart, controlling the direction and speed of rotation of the motor; the motor drives a micrometer screw which changes the yaw angle of the dihedral. This ensures that the science beam stays nominally centered on the relay optical elements, and enters the beam combination area without vignetting. Fine-scale correction is supplied automatically during star observations by the star tracker system.

We designed a trihedral mirror and mount subsystem, and fabricated the mechanical and optical components. The trihedral comprises an upper mirror unit and a lower mirror unit, both held in a stiff aluminum frame with appropriate fine-pitch adjusting screws. The lower mirror unit is a single flat. The upper mirror unit is a pair of flats, held at $90^{\circ}$ by a diagonal brace. The mirrors are Astrositall, and the brace is ULE. The mirrors are 1/20 wave peak to valley and silver coated (Denton FSS-99), similar to the other flat relay mirrors in IOTA.

Due to space constraints, the star beam is centered on the vertex line between the two upper mirror halves. In order to introduce no more than a $1 / 20$ wave peak to valley additional wavefront error from the error in the $90^{\circ}$ angle between these mirror halves, the tilt of the reflected wave segments must be no more than 0.4 arcsec. To achieve this accuracy we inserted the mirror segment assembly in one arm of an interferometer, and adjusted the angle until straight fringes were achieved.

We then glued the segments in place using the diagonal brace and 2 small supporting right-angle prisms along the back edge of the hinge line between segments. The glue is an ultra-violet light setting cement (Norland 61) commonly used for optical assemblies. We found that as it cured, over a time interval of roughly 10 to $100 \mathrm{sec}$, depending upon the UV light intensity, the cement changed dimension by a small but non-negligible amount. The result was that our included angle became acute, by an amount sufficient to make the straight-fringe interferogram turn into a chevron shape, with nominally one fringe of error at the edge of the assembly.

Subsequent inquiry suggests that this cement and others like it all experience a small shrinkage when cured, a behavior which is consistent with the observed change of angle. We have tried many variations of this experiment and always end up with the same or worse result. We are sure that the problem arises because of glue shrinkage. Other effects, such as thermal drifts and heating of the glass materials by the UV lamp, are demonstrated to be much smaller.

We believe that the mirrors must be rigidly bonded by cement in order to preserve the $1 / 20$ wave criterion, and in particular that they cannot be held by an external metal framework. Our failure to achieve the desired accuracy 
is a design challenge for the future. But in order to continue to make progress with the third telescope project, we have decided to abandon trihedrals for the near future, and instead use the dihedrals which have served us well for several years now, albeit with the nuisance of having to make a yaw correction every time the long delay mirror is moved.

\section{THREE-BEAM COMBINATION}

We have several paths that we can follow to achieve the 3-beam combination required for phase-closure measurements. Each of the paths mentioned here is currently being pursued.

For the infrared JHK bands we will build a classical Michelson beam-combiner assembly, using flat-plate combiners. For the infrared $\mathrm{H}$ band we will install an integrated optics beam combiner, currently being developed at Grenoble Observatory ${ }^{9}$; this project will be led by J.-P. Berger. For the visible, we are starting to experiment with visible-wavelength single-mode fibers, first for 2 beam combination, and later with 3 beams. These projects will be reported in future publications.

\section{PICNIC CAMERA}

Our project to upgrade our NICMOS3 camera $^{10}$ to a lower read-noise PICNIC chip is well underway, but not yet complete. We plan to build 2 such detector units, so that the second can be used as an infrared star tracker for sources with low visible-wavelength signals.

\section{FRINGE PACKET TRACKING}

Progress toward fringe tracking at IOTA is reported in this volume by Morel. ${ }^{7}$ The basic idea is that we are trying to merely keep the interference fringe packet centered in our scan window, to an accuracy of about one wavelength, for the purpose of being able to have the fringe packets in the 3-telescope system all be recorded at nearly the same instant of time. It is not necessary to control the piston to much greater accuracy for phase closure. The packet tracker has been successfully tested in preliminary experiments at IOTA, and it is currently being reformulated for inclusion in the VxWorks control system, where its implementation will be much simpler than is presently possible.

We are grateful to NASA for funding (NAG5-4900) the hardware required for the 3rd telescope upgrade at IOTA, and for supporting the fringe-packet tracking work for IOTA at SAO and NASA-Ames. We also gratefully acknowledge ongoing institutional support from the Smithsonian Astrophysical Observatory and the University of Massachusetts at Amherst.

\section{REFERENCES}

1. W. A. Traub, "Recent results from the IOTA interferometer," in Proc. SPIE 3350: Astronomical Interferometry, R. D. Reasenberg and J. B. Breckinridge, eds., p. 848, 1998.

2. J.-P. Berger, F. Malbet, R. Millan-Gabet, W. Traub, and M. Colavita, "Insights in the nature of the sub-AU circumstellar environment of FU Orionis," in Proc. SPIE 4006: Interferometry in Optical Astronomy, P. Lena and A. Quirrenbach, eds., SPIE, 2000.

3. K. Hofmann, T. Bloecker, V. C. du Foresto, M. Lacasse, S. Morel, B. Pras, D. Schertl, M. Scholz, W. Traub, , G. Weigelt, and M. Wittkowski, "Observations of Mira stars with the IOTA interferometer," in Proc. SPIE 4006: Interferometry in Optical Astronomy, P. Lena and A. Quirrenbach, eds., SPIE, 2000.

4. P. Kervella, V. C. du Foresto, W. Traub, and M. Lacasse, "Cepheid observations by long-baseline interferometry with FLUOR/IOTA," in Proc. SPIE 4006: Interferometry in Optical Astronomy, P. Lena and A. Quirrenbach, eds., SPIE, 2000.

5. B. Mennesson, G. Perrin, G. Chagnon, V. C. du Foresto, S. Morel, C. Ruilier, W. Traub, N. Carleton, and M. Lacasse, "Thermal infrared stellar interferometry using single-mode guided optics: first scientific results on IOTA," in Proc. SPIE 4006: Interferometry in Optical Astronomy, P. Lena and A. Quirrenbach, eds., SPIE, 2000.

6. R. Millan-Gabet, F. Schloerb, and W. Traub, "Investigation of Herbig Ae/Be stars in the near-infrared with the IOTA," in Proc. SPIE 4006: Interferometry in Optical Astronomy, P. Lena and A. Quirrenbach, eds., SPIE, 2000. 
7. S. Morel, W. Traub, J. Bregman, R. Mah, and E. Wilson, "Fringe-tracking experiments at the IOTA interferometer," in Proc. SPIE 4006: Interferometry in Optical Astronomy, P. Lena and A. Quirrenbach, eds., SPIE, 2000.

8. I. Porro, W. Traub, and N. Carleton, "Effect of telescope alignment on a stellar interferometer," Applied Optics 38, pp. 6055-6067, 1999.

9. F. Malbet, P. Kern, I. Schanen-Duport, J.-P. Berger, K. Rousselet-Perraut, and P. Benech, "Integrated optics for astronomical interferometry," Astron. Astrophys. Suppl. 138, pp. 135-145, 1999.

10. R. Millan-Gabet, F. P. Schloerb, W. A. Traub, and N. P. Carleton, "A NICMOS3 camera for fringe detection at the IOTA interferometer," Publ.. Astron. Soc. Pac. 111, p. 238, 1999. 